\title{
Optimal Transmission Radius for Flooding in Large Scale Sensor Networks
}

\author{
Marco Zúñiga Z. and Bhaskar Krishnamachari \\ Department of Electrical Engineering, University of Southern California, \\ Los Angeles, CA 90089,USA. \\ E-mail: \{marcozun,bkrishna\}@usc.edu
}

\begin{abstract}
One of the principal characteristics of large scale wireless sensor networks is their distributed, multi-hop nature. Due to this characteristic, applications such as query propagation rely regularly on network-wide flooding. Besides consuming energy and bandwidth resources, the flooded packet may keep the transmission medium within the network busy for too long, reducing overall network throughput. We analyze the impact of the transmission radius on the average settling time - the time at which all nodes in the network finish transmitting the flooded packet. We show that for large wireless networks there exists a transmission range which minimizes the settling time - corresponding to an optimal tradeoff between reception and contention times.
\end{abstract}

\section{INTRODUCTION}

Sensor networks present new challenges due to their unique characteristics, one of them being their distributed, multi-hop, dynamic nature. Due to this characteristic, applications rely regularly on flooding as a robust way to reach all the nodes in the network. An example of these applications is query propagation in directed diffusion [2].

If the transmission radius of the nodes is not carefully chosen, the flooded packet may take too long to be transmitted by all the nodes in the network, impacting overall network throughput. This becomes a serious problem in large-scale sensor networks where hundreds or thousands of nodes are present. This paper addresses the problem of finding an optimal transmission radius for flooding in sensor networks in order to minimize the time that the channel is captured by the flooded packet.

The intuitive idea is that as the transmission radius increases, each node will be able to send a packet directly (one hop away) to more nodes, thus reducing the number of hops required to reach all nodes. On the other hand, a bigger transmission radius implies more neighbors competing to access the medium, and hence a longer contention delay for packet transmission. The purpose of our work is the analysis of this trade-off in MAC protocols based on the exponential back-off algorithm, such as IEEE 802.11.

In this study, we assume that the nodes are static, do not fail and that they are deployed in either a grid or a uniform topology in a square area. In a uniform topology, the physical terrain is divided into a number of cells based on the number of nodes in the network, and a node is placed randomly within each cell.

The remainder of the paper is organized as follows. Section
II positions our work in the literature, discussing other related work. Section III describes the analytical model that we formulate to obtain the optimal transmission radius in sensor networks. Section IV shows the results obtained through simulations. Finally, Section V describes the conclusions and future work.

\section{RELATED WORK}

In recent years, sensor networks have engaged the attention of the research community due to their multiple applications, ranging from environmental sensing, structural monitoring, and industrial process control to emergency response and mobile target tracking. In [1], Akyildiz et al. present a survey of protocols and algorithms to date. Many of these protocols rely on flooding to disseminate information in the network.

Even though flooding has some unique advantages - it guarantees that all reachable nodes inside a network will receive the packet - it has several disadvantages as well. The broadcast storm paper by $\mathrm{Ni}$ et al. [3] has addressed how harmful this phenomenon can be. In that work, the authors study the broadcast storm problem in the context of wireless mobile ad-hoc networks, these networks consists of tens or hundreds of nodes. The broadcast storm problem is aggravated by the fact that there might be thousands of nodes in largescale sensor networks.

Other studies have looked at the impact of the transmission range in wireless networks. In [5] and [8] the authors analyze the critical transmission range to maintain connectivity in wireless networks, [5] presents an statistical analysis and [8] provides an algorithm for maintaining connectivity with some probability. On the same line of work, [6] and [7] analyze the minimum number of neighbors that a node should have to keep the network connected. Krishnamachari et al. [11] discuss critical transmission ranges for other global network properties such as Token Ring formation and channel allocation.

In [10], the authors also use the trade-off due to the increase of the transmission radius: a short range implies less collision and a long range implies moving a packet far ahead in one hop. However, in that work the authors want to maximize a parameter called the expected one-hop progress in the desired direction, which measures how fast a packet reaches its destination.

It is important to remark that all these previous studies were not analyzing a protocol like flooding, but trying $t$ 


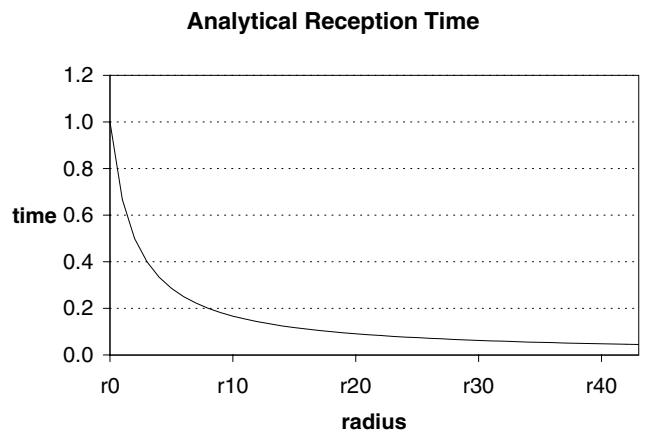

Fig. 1. Analytical result for reception time

an optimal transmission range for graph-based metrics such as connectivity. [4] investigates the impact of link layer nonidealities on the dynamic behavior of flooding. In that work, an experimental testbed of 150 rene motes [9] run flooding as the routing protocol. The study showed empirical relations between the reception and settling times - parameters used in our work - for different transmission ranges.

Our work also tries to find an optimal radius. However, in our case the important metric is the amount of time that a flooded packet captures the transmission medium. This metric is of great importance in large scale wireless networks because of its relation to the throughput: the less time the channel is captured by a flooding event, the more queries can be disseminated and the more time the channel is available for data packets transmission.

We can state the relation between settling time and throughput in sensor networks as follows:

$$
\text { throughput } \alpha \frac{1}{\text { settlingtime }}
$$

To accomplish our goal - minimizing the settling time - we study the tradeoff between reception and contention times.

\section{ANALYTICAL MODEL}

Our analytical model is based on the following definitions:

- Reception Time ( $T_{R}$ ): Average time when all the nodes in the network have received the flooded packet

- Contention Time ( $T_{C}$ ): Average time between reception and forwarding (delivery) of a packet by all the nodes in the network.

- Settling Time ( $T_{S}$ ): Average time when all the nodes in the network have forwarded (delivered) the flooded packet.

From these definitions we observe that:

$$
T_{S}=T_{R}+T_{C}
$$

The purpose of our study is to determine an optimal transmission radius that minimizes the settling time. Since the settling time is the sum of the reception and contention times,
Fig. 2. Different regions to calculate the number of neighbors as we increase the transmission radius of the node. The first region is for values of $\mathrm{R}$ between 0 and $R_{e}$. The second region goes from $R_{e}$ to $R_{w}$; in this region we can observe that some parts of the coverage area does not contribute with more nodes. In the third region -when $\mathrm{R}$ is greater than $R_{w}$ - all the nodes in the network are neighbors.

the remaining of this section will analyze the relation between $T_{R}$ and $T_{C}$ with the length of the transmission radius.

Let us first consider the relation between the transmission radius and the reception time. If the transmission radius of each node is increased, the reception time in the network will decrease. The two extreme cases are as follows:

- The nodes have a minimum radius that guarantees a connected network.

- The nodes have a radius that covers the whole network.

In the first case, the number of hops required between the source and the last node to receive the packet is maximum and hence the reception time will increase. In the second case, all the nodes will be one hop away from the source, and hence all of them will receive the packets at almost the same time.

Now, let us consider the relation between the transmission radius and the contention time. If a node increments its transmission radius, it will increase its number of neighbors (degree of the node), which will subsequently increase the contention time. We will consider again the two extreme cases to visualize this effect. If the transmission radius is the minimum that guarantees the connectivity of the network, each node will have the minimum number of neighbors and thus the node will face a minimum contention time when transmitting the packet. On the other hand, when the transmission radius covers the whole network, all the nodes in the network will compete for accessing the medium, thus increasing their contention time.

To summarize this discussion, we state that as the transmission radius increases, the reception time decreases and the contention time increases. Since equation (2) shows that the settling time is the sum of these quantities, the minimum settling time will correspond to some intermediate radius. We now analyze this phenomenon mathematically.

\section{A. Reception Time}

The reception time $T_{R}$ is going to be directly proportional to the distance between the transmitter and the rece: 
Average Number of Neighbors 500 nodes, $1000 \times 1000 \mathrm{~m}^{2}$ area

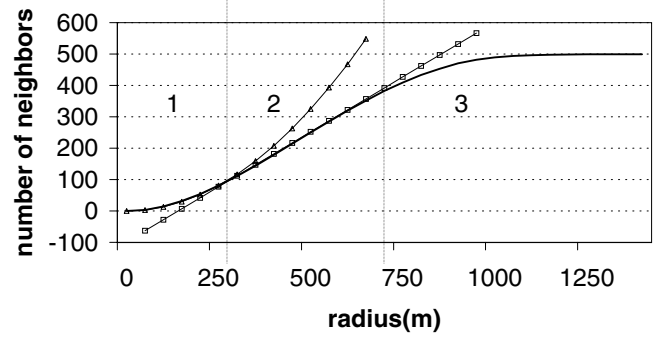

Fig. 3. Average number of neighbors for 500 nodes in a 1000x1000 area

inversely proportional to the transmission radius. Due to the kind of topologies considered in our research (grid or uniform), the distance between nodes is proportional to the length of the side of the area. Let us define:

$\mathrm{R}$ : transmission radius.

$\mathrm{S}$ : length of the side of the square area.

Then we have that

$$
T_{R} \alpha \frac{S}{R}
$$

Figure 1 shows the analytical result for the reception time. In this figure we are interested in the trend of the curve and for that reason the plot does not show any units.

More accurately, $T_{R}$ is not only proportional to the distance between the transmitter and the receiver, and inversely proportional to the transmission radius. But if the flooding operation needs more than one hop to reach all nodes, $T_{R}$ will also depend on the contention time faced at each hop. However, we are interested in the first time the flooded packet is received by the nodes and for this reason we are primarily interested in the first packet that is retransmitted by a set of contending nodes. The first successful packet in this contention will not have a significant delay. Hence, we do not consider the contention time in equation (3).

\section{B. Contention Time}

The contention time $T_{C}$ is a function of the number of neighbors. If we consider the area covered by the network as $S^{2}$, then the expected number of neighbors of a given node is:

$$
\tilde{m}=\pi \frac{R^{2}}{S^{2}} n
$$

Where $\mathrm{n}$ is the total number of nodes in the network.

The contention time is not directly proportional to the equation given in (4). There are two effects that influence $T_{C}$; we call them edge effect and exponential back off effect. Both effects are described below.
Contention Time vs Number of Neighbors

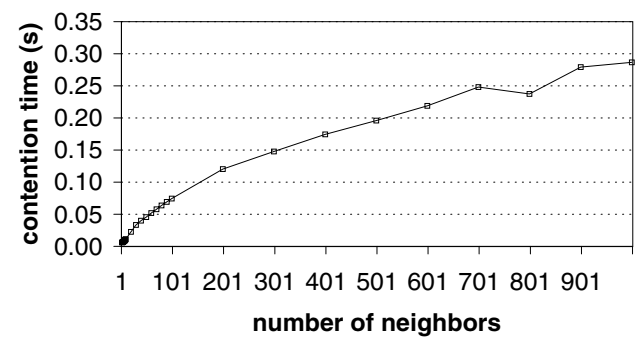

Fig. 4. Exponential back off effect:A we can observe that the relation between the contention time and the number of neighbors is not linear

1) Edge Effect: The edge effect can be described as follows; nodes on the edge will not increase their number of neighbors proportionally to the square of the radius. The reason is that only a fraction of the area covered by its transmission range intersects the area of the topology. This phenomenon is illustrated in Figure 2, which shows a square topology with the source node placed in the intersection of two lines. In this figure we can observe three regions as the transmission radius is increased.

- Region 1: When $\mathrm{R}$ goes from 0 to the closest distance of the source node to any edge $\left(R_{e}\right)$.

- Region 2: When $\mathrm{R}$ goes from the closest distance of the source node to any edge $\left(R_{e}\right)$, until it covers the whole network $\left(R_{w}\right)$.

- Region 3: When $\mathrm{R}$ is greater than the value that covers the whole network $\left(R_{w}\right)$.

Figure 3 shows the curve for the average number of neighbors versus the transmission radius, for a topology of 500 nodes in a $1000 \times 1000 \mathrm{~m}^{2}$ area. To obtain this curve, we calculate the number of neighbors for each node in the topology and for different values of transmission radius. Then, for each value of these transmission radius we take the average number of neighbors for all the nodes in the network.

In figure 3 , it is clear to observe the three regions described in figure 2 - plots of a quadratic curve and a liner curve were added to visualize the first two regions. Each of these regions (1, 2 and 3) will have a different expression for the number of neighbors. For the first region, the number of nodes under the transmission radius is directly proportional to the square of the transmission radius. In the second region, the number of neighbors increases proportionally to the overlapping area between the transmission range and the network area. We will approximate this region by a linear relation. In the third region, the number of neighbors remains constant and is equal to the total number of nodes in the network.

Finally, we obtain that the average number of neighbors $m$ due to the edge effect is: 


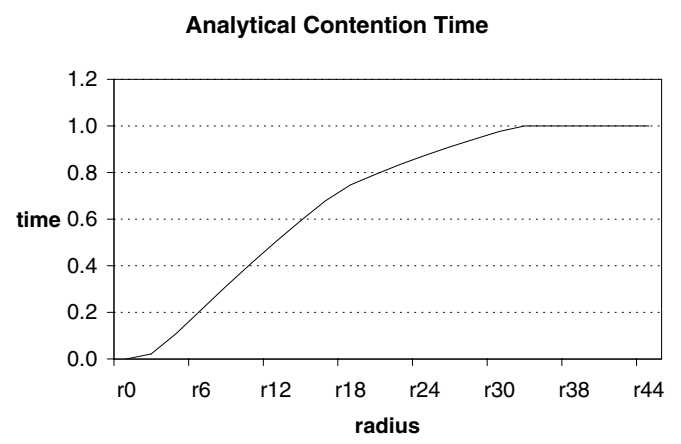

Fig. 5. Analytical contention time

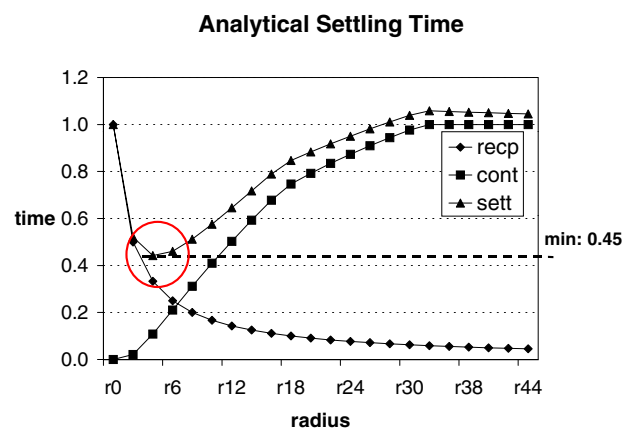

Fig. 6. Analytical settling time

$$
m= \begin{cases}\pi \frac{R^{2}}{S^{2}} n & , 0<R<R_{e} \\ \frac{R}{S} n & , R_{e}<R<R_{w} \\ n & , R_{w}<R\end{cases}
$$

It is important to observe that in some realistic scenarios, large scale wireless sensor networks may not be able to reach regions 2 and 3 due to the large transmission range $R$ required. However, since we do not have a prior knowledge of in what region the optimal radius is, we need to take into account all the possible cases in the mathematical model. In section III-C further analysis establishes that the optimal radius is in region 1 .

2) Exponential Back Off Effect: In IEEE 802.11, a node checks if the medium is clear before sending a packet; when the medium is clear for a small period of time (DIFS), the node transmits the packet. If the channel becomes busy, it will back off exponentially (in order to avoid congestion) to transmit the packet. This mechanism, known as CSMA/CA, leads to a non-linear relation between the contention time versus the number of neighbors. We denote this non-linear relation as the exponential back off effect.

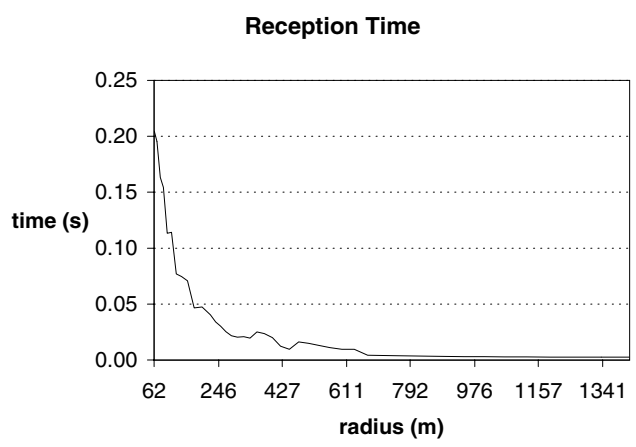

Fig. 7. Reception time obtained through simulations for 500 nodes in a $1000 \times 1000 \mathrm{~m}^{2}$ area

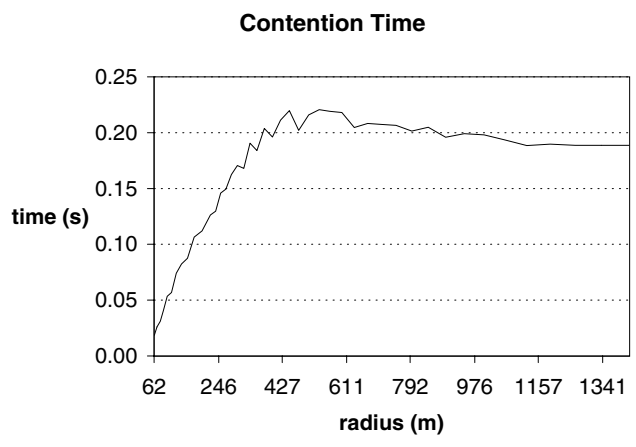

Fig. 8. Contention time obtained through simulations for 500 nodes in a $1000 \times 1000 \mathrm{~m}^{2}$ area

Figure 4 shows this non-linear relation obtained by simulations as follows: a wireless network is created where all the nodes are within the transmission range of any other node (i.e. one hop away). One node floods a packet and the settling time is measured. This experiment was done for different number of nodes in the network.

The curve in figure 4 can be numerically approximated by:

$$
f(m)=J \log ^{2}(m)
$$

Where $\mathrm{m}$ is the number of neighbors and $\mathrm{J}$ is a constant.

If we incorporate the two effects explained above, we obtain that the contention time $T_{C}$ is given by:

$$
T_{C}= \begin{cases}K f\left(\pi \frac{R^{2}}{S^{2}} n\right) & , 0<R<R_{e} \\ L f\left(\frac{R}{S} n\right) & , R_{e}<R<R_{w} \\ M n & , R_{w}<R\end{cases}
$$

Where $\mathrm{K}, \mathrm{L}$, and $\mathrm{M}$ are constants and $f($.$) is the function$ described in equation (6).

Figure 5 shows the analytical curve for the content 


\begin{tabular}{|l|c|c|c|c|}
\hline Area side $(m)$ & 500 nodes & 600 nodes & 700 nodes & 800 nodes \\
\hline 1000 & 125.2 & 198.5 & 140.5 & 157.7 \\
\hline 2000 & 251.8 & 237.7 & 251.8 & 222.7 \\
\hline 3000 & 335.8 & 355.7 & 282.5 & 355.7 \\
\hline 4000 & 422.8 & 474.3 & 447.8 & 376.8 \\
\hline 5000 & 474.3 & 474.3 & 447.8 & 502.4 \\
\hline
\end{tabular}

TABLE I

OPTIMAL RADIUS OBTAINED FOR DIFFERENT TOPOLOGIES IN METERS

\begin{tabular}{|l|c|c|c|c|}
\hline Area side $(m)$ & 500 nodes & 600 nodes & 700 nodes & 800 nodes \\
\hline 1000 & $35 \%$ & $33 \%$ & $38 \%$ & $35 \%$ \\
\hline 2000 & $45 \%$ & $52 \%$ & $55 \%$ & $49 \%$ \\
\hline 3000 & $47 \%$ & $50 \%$ & $53 \%$ & $52 \%$ \\
\hline 4000 & $40 \%$ & $44 \%$ & $48 \%$ & $52 \%$ \\
\hline 5000 & $38 \%$ & $49 \%$ & $50 \%$ & $52 \%$ \\
\hline
\end{tabular}

TABLE II

PERCENTAGE OF THE MAXIMUM IMPROVEMENT THAT CAN BE OBTAINED IN THE SETTLING TIME

As in figure 1, we are interested in the trend of the curve and not the units.

\section{Settling Time}

Considering the different regions in the contention time due to edge effects, the settling time $T_{S}$ - defined as the sum of the reception and contention times - is given by:

$$
T_{S}= \begin{cases}k_{1} \frac{S}{R}+K f\left(\pi \frac{R^{2}}{S^{2}} n\right) & , 0<R<R_{e} \\ k_{1} \frac{S}{R}+L f\left(\frac{R}{S} n\right) & , R_{e}<R<R_{w} \\ k_{1} \frac{S}{R}+M n & , R_{w}<R\end{cases}
$$

Figure 6 shows the analytical settling time. For illustrating purposes, the analytical receiving and contention times were also plotted. It is important to observe that according to our model, we can obtain improvements up to $55 \%$ in the settling times.

The settling time curve shows a minimum, as expected. Furthermore, the curve shows that the minimum is in either regions 1 or 2 according to figure 3 , allowing us to restrict our analysis to those two regions.

In order to obtain the optimal minimum, we take the derivative respect to $\mathrm{R}$ of the settling time obtained in (8) and set it to zero. Some basic mathematical manipulation leads to the following expressions in regions 1 and 2 respectively:

$$
\begin{aligned}
& k_{1} \frac{S}{R}=4 K \log \left(\pi \frac{R^{2}}{S^{2}} n\right) \log (e) \quad, 0<R<R_{e} \\
& k_{1} \frac{S}{R}=2 \log \left(\frac{R}{S} n\right) \log (e) \quad, R_{e}<R<R_{w}
\end{aligned}
$$

In each region there is an $\mathrm{R}$ corresponding to the solution of these equations. The optimum $\mathrm{R}$ is one of these two radii (which offers the minimum $T_{S}$ ). The constants $k_{1}, K$ and $L$ will be obtained through simulations.

\section{Simulation Set Up and Results}

This section describes the set up of the simulations and the results obtained.

\begin{tabular}{|l|c|c|c|c|}
\hline Area side $(m)$ & 500 nodes & 600 nodes & 700 nodes & 800 nodes \\
\hline 1000 & 0.0112 & 0.0107 & 0.0127 & 0.0135 \\
\hline 2000 & 0.0102 & 0.0102 & 0.0104 & 0.0107 \\
\hline 3000 & 0.0085 & 0.0093 & 0.0100 & 0.0107 \\
\hline 4000 & 0.0083 & 0.0095 & 0.0101 & 0.0093 \\
\hline 5000 & 0.0086 & 0.0094 & 0.0099 & 0.0097 \\
\hline
\end{tabular}

TABLE III

CONSTANT $k_{1}$ OBTAINED FOR DIFFERENT TOPOLOGIES

\begin{tabular}{|l|c|c|c|c|}
\hline Area side $(m)$ & 500 nodes & 600 nodes & 700 nodes & 800 nodes \\
\hline 1000 & 0.0031 & 0.0031 & 0.0032 & 0.0031 \\
\hline 2000 & 0.0022 & 0.0022 & 0.0024 & 0.0023 \\
\hline 3000 & 0.0018 & 0.0020 & 0.0019 & 0.0020 \\
\hline 4000 & 0.0017 & 0.0016 & 0.0017 & 0.0017 \\
\hline 5000 & 0.0017 & 0.0016 & 0.0017 & 0.0016 \\
\hline
\end{tabular}

TABLE IV

CONSTANT $K$ OBTAINED FOR DIFFERENT TOPOLOGIES

\section{A. Simulation Set Up}

In order to corroborate our analytical model we simulated different size of networks in terms of area and number of nodes. The simulation was done using the Global Mobile Simulation (GloMoSim) developed at UCLA [12]. The MAC layer used was IEEE 802.11 with a bandwidth of $2 \mathrm{Mbps}$, since flooding only broadcast messages, IEEE 802.11 behaved like CSMA/CA (i.e. no RTS/CTS nor ACK).

Algorithm 1 shows the stages at which we stored $T_{R}, T_{C}$ and $T_{S}$

\section{Algorithm 1: Flooding}

if message received for the first time then

Save receiving time; // used to calculate $T_{R}$

Rebroadcast Packet;

Save delivery time; // used to calculate $T_{C}$ and $T_{S}$

First we create a uniform topology placing $\mathrm{n}$ number of nodes in a $S \times S$ square area. All the nodes in the network have the same transmission range $\mathrm{R}$. Then, we run breadth first search and binary search algorithms to obtain the minimum radius that connects the network. Starting with that radius, we increase the transmission radius of the nodes until it covers the whole network. The source node is placed at the bottom left corner of the area, hence the maximum transmission radius is set to $R=S \sqrt{2}$.

The source sends a packet of fixed size (512 bytes). Usually queries or raw data packets in sensor networks will be smaller than that. We believe that the packet size chosen is a reasonable upper bound.

\begin{tabular}{|l|c|c|c|c|}
\hline Area side $(m)$ & 500 nodes & 600 nodes & 700 nodes & 800 nodes \\
\hline 1000 & 131.5 & 123.5 & 131.2 & 136.2 \\
\hline 2000 & 307.9 & 298.2 & 277.8 & 284.5 \\
\hline
\end{tabular}

TABLE V

ANALYTICAL RADIUS FOR FIRST REGION IN METERS 


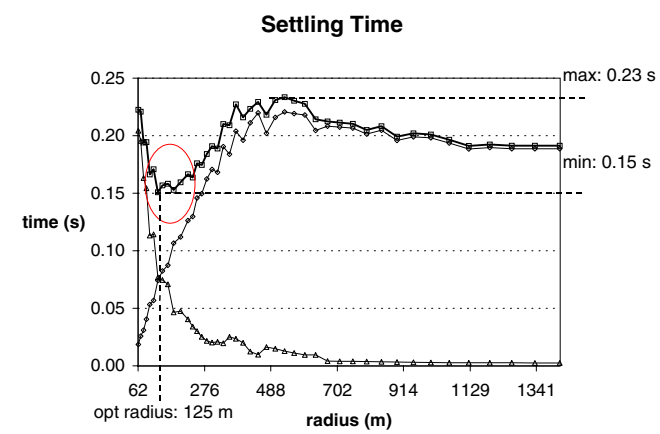

Fig. 9. Settling time obtained through simulations for 500 nodes in a $1000 \times 1000 \mathrm{~m}^{2}$ area

\section{B. Results}

Figure 7 shows the reception time for 500 nodes in a $1000 \times 1000 \mathrm{~m}^{2}$ area. We observe that the reception time decreases until the point where the transmission radius covers all the nodes in the network. The plot resembles closely the one obtained in the analytical model (figure 1).

Figure 8 shows the contention time for the same topology. As expected, the contention time shows the edge effect in the network. This figure also resembles the analytical plot (figure $5)$.

The settling time is showed in figure $9-$ note similarity to figure 6 . We observe that an optimal transmission radius can be obtained. In this case the radius is approximately 125 meters. It is important to observe that the maximum settling time is around 0.23 seconds and the minimum is around 0.15 seconds. These results implies that the improvement obtained in the settling time is approximately $35 \%$. Table II shows that the improvement obtained for different topologies ranges between $40 \%$ and $50 \%$ - our analytical model showed a maximum improvement of $55 \%$ (figure 6).

Table I shows the optimal radius obtained for different topologies. One obvious observation is that as we increase the area of the network - keeping the number of the nodes constant - the optimal transmission radius also increases.

One important step in the simulations is to obtain the value of the constants that can be used to compute the analytical optimal radius. Tables III and IV shows the values for $k_{1}$ and $K$ respectively. In all these constants we observe some dependency on the side of the area. However, as the area of the network is increased, all the constants tend to converge to a single value. The reason for this trend is the influence that the packet size has in the reception and contention times in small-area networks. Due to the lack of space, we can not discuss the details of this behavior.

According to the regions specified by the edge effect in section III-B (figure 3), we can observe from figure 9 that the optimal radius is in region 1 , since the minimum settling time is obtained for a radius less than $300 \mathrm{~m}$.
By replacing the constants obtained in tables III and IV in equation (9), we obtain that the analytical minimum radius are fairly close to the ones obtained in the simulations, as shown in table $\mathrm{V}$.

\section{Conclusions And Future Work}

This paper addresses the idea of finding an optimal transmission range for flooding in sensor networks. Discussing this problem in sensor networks is of great importance due to flooding usefulness in applications like query propagation.

There is significant work in the literature that has studied optimal transmission ranges for maintaining network connectivity and to make efficient use of energy in the network. To the best of our knowledge, finding an optimal transmission radius to minimize the settling time in flooding events has not been addressed in depth before.

This work proves that choosing a transmission radius without previous analysis can lead to an unnecessarily large settling time in the network which will decrease the overall network throughput. We propose a mathematical model - supported by simulations - to find an optimal radius for uniform and grid topologies. Improvements between $30 \%$ and $55 \%$ can be achieved in the settling time as shown in table II.

Finally, this work was based considering only uniform or grid topologies. Future work will analyze this problem on other kinds of deployments as well as other MAC schemes.

\section{REFERENCES}

[1] Akyildiz, I.F., Su, W., Sankarasubramaniam, Y., and Cayirci, E., "A Survey on Sensor Networks, IEEE Communications Magazine," Vol. 40, No. 8, pp. 102-116, August 2002.

[2] Chalermek Intanagonwiwat, Ramesh Govindan and Deborah Estrin, "Directed diffusion: A scalable and robust communication paradigm for sensor networks" In Proceedings of the Sixth Annual International Conference on Mobile Computing and Networking, August 2000.

[3] S. Ni, Y. Tseng, Y. Chen, and J. Chen, "The broadcast storm problem in a mobile ad hoc network," In Annual ACM/IEEE International Conference on Mobile Computing and Networking, pp. 151-162, August 1999.

[4] Deepak Ganesan, Bhaskar Krishnamachari, Alec Woo, David Culler, Deborah Estrin and Stephen Wicker, "Complex Behavior at Scale: An Experimental Study of Low-Power Wireless Sensor Networks", UCLA Computer Science Technical Report UCLA/CSD-TR 02-0013

[5] P. Gupta and P. R. Kumar, "Critical Power for Asymptotic Connectivity in Wireless Networks," in Stochastic Analysis, Control, Optimization and Applications, Eds.W.M.McEneany et al., Birkhauser, Boston, 1998.

[6] Feng Xue and P. Kumar, "The number of neighbors needed for connectivity of wireless networks." Wireless Networks. April, 2002.

[7] L. Kleinrock and J . A. Silvester. "Optimum transmission radii for packet radio networks or why six is a magic number" in Conf. Rec. Nat. Telecommun. Conf., Dec. 1978, pp. 4.3.1-4.3.5.

[8] M. Sanchez , P. Manzoni and Z. Haas, "Determination of Critical Transmission Range in Ad-Hoc Networks," Multiaccess Mobility and Teletraffic for Wireless Communications Workshop, Italy, October 1999.

[9] TinyOS Homepage. http://webs.cs.berkeley.edu/tos/

[10] Takagi, H. and L. Kleinrock, "Optimal Transmission Ranges for Randomly Distributed Packet Radio Terminals", IEEE Transactions on Communications, Vol. COM-32, No. 3, pp. 246-257, March 1984.

[11] Bhaskar Krishnamachari, Stephen Wicker, Ramon Bejar, and Marc Pearlman, "Critical Density Thresholds in Distributed Wireless Networks," book chapter in Communications, Information and Network Security, eds. H. Bhargava, H.V. Poor, V. Tarokh, and S. Yoon, Kluwer Publishers, December 2002.

[12] http://pcl.cs.ucla.edu/projects/glomosim/ 